\title{
Voronoï imaging methods for the measurement of granular flows
}

\author{
H. Capart, D. L. Young, Y. Zech
}

\begin{abstract}
A set of digital imaging methods derived from the Voronoï diagram is proposed and tested on various liquid-granular flow applications. The methods include a novel pattern-based particle-tracking algorithm, as well as estimators of the three-dimensional granular concentration from two-dimensional images. The proposed algorithms are able to resolve individual grain motions even for rapid shear flows involving dense, fluctuating granular ensembles. Full automation is achieved, allowing the derivation of accurate statistics from large sets of individual measurements, as well as the construction of complete sets of grain trajectories. Results are presented for different applications: homogeneous fluidization, steady uniform debris flow, and unsteady debris surges.
\end{abstract}

\section{1}

\section{Introduction}

Flows of granular materials are notable for the diversity of their behaviours and their involvement in a wide range of geophysical and industrial processes. While much about

Received: 12 December 1999 /Accepted: 21 June 2001

Published online: 29 November 2001

H. Capart ( $\varangle)$

Fonds National de la Recherche Scientifique and

Department of Civil Engineering,

Université catholique de Louvain,

1 place du Levant, 1348 Louvain-la-Neuve, Belgium

D. L. Young

Department of Civil Engineering and Hydrotech Research

Institute, National Taiwan University,

158 Chow Shan Rd, Taipei 10617, Taiwan

Y. Zech

Department of Civil Engineering,

Université catholique de Louvain,

1 place du Levant,

1348 Louvain-la-Neuve, Belgium

The fluidization cell tests were performed at the Laboratoire du Génie Civil, Université catholique de Louvain, Belgium. The uniform debris flow experiments were conducted by L. Guarino at the Hydraulics Laboratory of the Civil and Environmental Engineering Department of the Università degli Studi di Trento, Italy. H. C. benefited there from the hospitality and support of professors A. Armanini and L. Fraccarollo. The dam-break measurements were obtained with the help of Y.H. Liu at the Hydrotech Research Institute, National Taiwan University, Taiwan, and with support of a travel grant from the Fonds National de la Recherche Scientifique, Belgium. them can be learned from computational simulations (Campbell 1990; Kalthoff et al. 1997), there is a corresponding need for detailed experimental measurements. A variety of techniques are available for multiphase flow measurements (Bachalo 1994; Chaouki et al. 1997), and those applied more specifically to granular flows include force and impact measurements (Savage and McKeown 1983; Zenit et al. 1997), acoustic probes (Bennett and Best 1995), tracked transmitters (Dave et al. 1999), and magnetic resonance imaging (Nakagawa et al. 1993). The present work focuses on digital imaging techniques applied to the analysis of monocular image sequences. These are typically acquired by filming a flow free-surface from above or by imaging the flow through a transparent wall. As granular flows are characterized by high solid fractions, opacity of the material generally prevents optical penetration beyond a few grain diameters. In many instances, this does not prevent one from making meaningful measurements, and it is not necessary to resort to sophisticated techniques such as magnetic resonance imaging or refractive-index matching (Cui and Adrian 1997) to penetrate the flow interior. One would therefore wish to resort to the powerful imaging techniques of experimental fluid mechanics (Adrian 1991) to provide nonintrusive, whole-field coverage of the flow kinematics of the visible grains.

Granular flows are, however, characterized by three features that pose problems to imaging velocimetry algorithms: dense dispersions of grains, fluctuating motions produced by interparticle contacts, and sharp flow gradients on the scale of only a few grain diameters. The simplest methods of particle-tracking velocimetry (PTV) rely on minimum displacement matching (Guler et al. 1999) and fail when the interframe displacement becomes significant with respect to the mean particle interdistance. This limit is quickly reached for dense, rapid granular flows. More sophisticated particle-tracking methods involving trajectory-based matching (Sethi and Jain 1987) are easily offset by the uncorrelated granular velocity fluctuations. The techniques of particle imaging velocimetry (PIV) are robust with respect to large displacements of dense dispersions (Willert and Gharib 1991) but have difficulties in dealing with intense shear (Huang et al. 1993). Finally, the direct-correlation (Fujita et al. 1998) or spatial filtering methods (Uddin et al. 1998) do not resolve individual grain motions, an information which is most important if one seeks to test microstructural theories.

Similar difficulties are encountered for concentration estimation. When grains become closely packed, occlusion 
effects hamper the correspondence between two-dimensional observations of the grain number density and their three-dimensional volumetric concentration. Suggestions have been made for such a correspondence (Capart et al. 1997), but have not been tested in detail. Proposals based on luminance measurements (Louge and Jenkins 1997; Kanda et al. 1999), on the other hand, require case-by-case calibration and a careful control of illumination, which is difficult to obtain even in laboratory conditions.

Various strategies have been adopted by researchers to cope with these difficulties. A first possibility is to diminish tracking problems by restricting the investigations to dilute dispersions or slow deformation rates, and to avoid concentration estimation problems by dealing with two-dimensional dispersions of spheres or disks moving between two closely spaced plates (Elliott et al. 1998; Wildman et al. 1999). Another widely used approach is to track only a small proportion of coloured "tracer" particles included in the dispersion (Natarajan et al. 1995; Liu et al. 1997; Hsiau and Jang 1998), leading to a corressponding loss of resolution. Finally, a third strategy consists in performing the tracking manually or with manual supervision (Drake 1991; Capart and Young 1998), limiting the number of measurements to the bounds of human patience.

In the present work, we develop and test imaging methods that are aimed at addressing the above shortcomings. Both for velocimetry and concentration, the methods derive from a pattern-based principle. The idea is that the local pattern formed by neighbouring grains will remain stable over a certain time, even for a rapidly deforming, fluctuating granular phase, and that it can therefore serve as a match template. Furthermore, it can also be used to characterize the local degree of packing of the dispersion. The specific tool chosen to describe local patterns is the Voronoï diagram. Various properties of the Voronoï diagram endow the approach with advantages over other pattern-based methods (Haynes and Turner 1992; Song et al. 1996, 1999; Ruan et al. 1999), and this will be discussed in detail in the presentation of the algorithms that forms the first part of the paper. In the second part, the methods are tested on selected liquidgranular flows, including homogeneous fluidization, steady uniform channel flow, and dam-break induced debris surges.

\section{2}

\section{Principle and algorithms}

Consider a sequence of images depicting the flow of an ensemble of grains. Rather than directly correlating windows of pixel values, the present work deals with grain images by first abstracting them into point-like particles. The granular ensemble is thus reduced to a set of featurepoints corresponding to grain centroids that are dispersed in the image plane. Particle identification can be achieved using a variety of segmentation or filtering methods, and the particular algorithm used for the applications is described in Appendix 1. After this operation, the analysis of the motions and patterns of sets of point-like particles constitutes the general object of the Voronoï methods.

\section{1}

\section{Voronoï construction and properties}

Let a dispersion of $n$ feature-points $P_{i}$ occupy positions $r_{i}=\left(x_{i}, y_{i}\right)(i=1, \ldots, n)$ in the two-dimensional plane (Fig. 1). The Voronoï construction designates the tiling of the plane into $n$ polygonal regions (or "cells") such that each polygon $V_{i}$ encompasses the region of the plane that is nearest to $P_{i}$ than to any other feature-point. Featurepoints characterized by Voronoï cells sharing an edge are termed "natural neighbours" of each other. The graph that connects natural neighbours further defines a second tesselation, dual to the Voronoï diagram: the Delaunay triangulation, composed of triangles $D_{j}$.

The two constructions present many remarkable properties that have led to recent applications in a variety of fields including cell biology (Marcelpoil and Usson 1992), computational mechanics (Braun and Sambridge 1995), astronomy (Bernardeau and van de Weygaert 1996), and molecular hydrodynamics (Espanol 1998). A general overview of the Voronoï diagram, its properties, and applications is given in Okabe et al. (1992), and a mathematical introduction in Preparata and Shamos (1985). In the present context, the Voronoï and Delaunay diagrams are of interest primarily because they provide useful local structures, or "tokens", which can be exploited for pattern characterization and matching (Ahuja 1982). The most obvious among these structures are the Voronoï cells and Delaunay triangles themselves, with properties such as area and perimeter.

Structures of a second type, most useful for patternmatching, define local "neighbourhoods". The triplets of feature-points that are vertices to a common Delaunay triangle provide one such local neighbourhood (Song et al. 1999). Another structure of this type, key to the present work, is the Voronoï 1-star (Fig. 2). The first vertex star (or simply 1-star) $S_{i}$ of feature-point $P_{i}$ is defined as the set of its natural neighbours, including itself (Senechal 1995). The 1-star can be visualized as a "star of spokes" originating at feature-point $P_{i}$. (Likewise, the 2-star of featurepoint $P_{i}$ can be defined as the set of feature-points that are natural neighbours to the 1-star of feature-point $P_{i}$, and so on). As shown in detail in the present work, the Voronoï 1-star constitutes a very useful token for pattern-matching in a particle-tracking context. A comparison with other neighbourhood definitions is provided in Sect. 2.2.

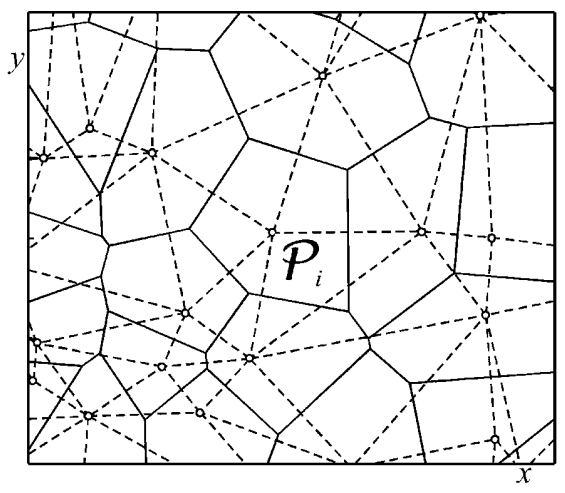

Fig. 1. Voronoï diagram $(-)$ and its dual, the Delaunay triangulation (- - ), constructed on a random dispersion of featurepoints $P_{i}(\mathrm{o})$ 


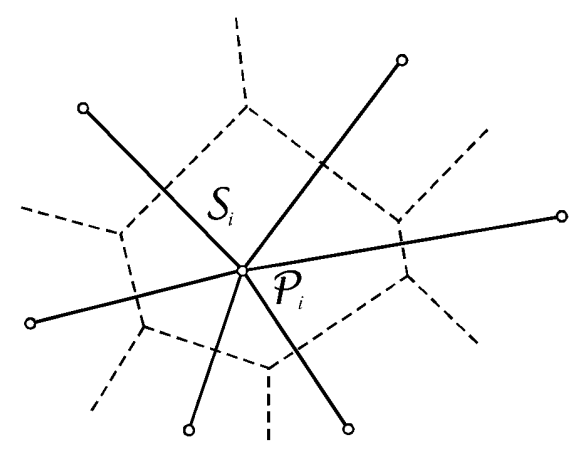

Fig. 2. The Voronoï 1-star $S_{i}(-)$ associated with one of the vertices $P_{i}(\mathrm{o})$ of the Voronoï diagram (- - -)

The Voronoï and Delaunay tesselations are characterized by the following desirable properties:

1) Geometric properties. First, except for unlikely degenerate cases, the construction is unique for a given set of feature-points. Secondly, the construction is local, i.e., the position of remote points does not affect the local structure of the diagram (Sibson 1981). The tokens described above can thus be construed as reliable descriptors of the local point pattern. Thirdly, the construction is adaptive with respect to variations in the local density of points. In particular, natural neighbours of a featurepoint tend to "surround" it evenly, regardless of a possible density gradient in one direction or another (Ahuja 1982).

2) Kinematic properties. The Voronoï diagram is stable to continuous deformation. This means that if the featurepoints move along continuous trajectories, then the shape of the Voronoï cell will also evolve gradually, and neighbourhood relations will change one by one (except for unlikely degenerate cases). If the trajectories of the feature-points are known, this can be used to continuously update the Voronoï diagram rather than reconstruct it from scratch at discrete times (Albers et al. 1998). This property makes the Voronoï 1-star a particularly attractive token for flowing dispersions of particles, as branches of the star can be expected to deform gradually, except for isolated topological events that will affect one branch of the star at a time. Delaunay triplets are not so attractive in this respect, since they are affected much more severely by these "swap" events (Fig. 3). The Voronoï diagram also remains stable in the case of addition or suppression of a feature-point, as in the case of particle occlusion.

3) Computational properties. The construction presents a number of desirable computational properties. First, algorithms are available that can construct the Voronoï diagram in $O(n \log n)$ operations (where $n$ is, as before, the number of feature-points). The particular algorithm used in the present work is based on Fortune's plane sweep method (Fortune 1987). Secondly, once the construction is obtained, a nearest-neighbour search (useful for matching operations) can be performed in $O(\log n)$ operations (Preparata and Shamos 1985). Finally, local reconstruction of the Voronoï diagram (after removal of a spurious particle image, for example) can be performed in $O(1)$ operations. These features make it possible to devise efficient implementations of the algorithms detailed in Sect. 2.2.
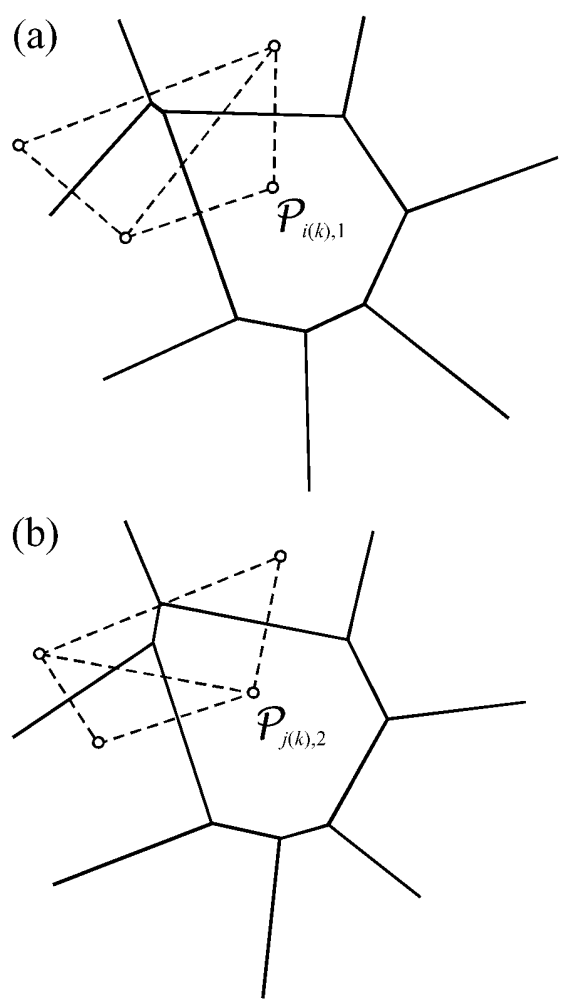

Fig. 3a, b. Local Voronoï diagram (-) and Delaunay triangles $\left(-\right.$ - -) constructed on a deforming set of points $(0)$ at times a $t_{1}$ and $\mathbf{b} t_{2}$. Because of the deformation, "swap" events may change the local configuration. Delaunay triangles are much more strongly affected by these topological changes than the Voronoï cells (and their associated 1-stars)

\section{2}

\section{The matching problem}

Consider now a set of moving particles, with positions sampled on two image frames acquired at instants $t_{1}$ and $t_{2}$. Supposing that particle images are indistinguishable from each other (particles are of the same size, colour, etc.), the information available is limited to the two sets of feature-point positions $r_{i, 1}\left(i=1, \ldots, n_{1}\right)$ and $r_{j, 2}(j=1, \ldots$, $n_{2}$ ). Based on this information, the objective is to match particles between the two frames. Formally, one seeks a pairing $\{i(k), j(k)\}$ associating particle image $P_{i}$ on frame 1 and particle image $P_{j}$ on frame 2 to one and the same physical particle $k$. Particle velocity vectors can then be obtained from:

$\boldsymbol{v}_{k}=\frac{\boldsymbol{r}_{j(k), 2}-\boldsymbol{r}_{i(k), 1}}{t_{2}-t_{1}}$

If the time interval $\Delta t=t_{2}-t_{1}$ is small enough, then a reasonable algorithm is to associate to each feature-point $P_{i, 1}$ of frame 1 the nearest feature-point of frame 2 . This is the "minimum displacement" algorithm (Jähne 1995; Guler et al. 1999), which can be written formally

$\operatorname{match}\left(P_{i, 1}\right)=\min _{P_{j, 2}}\left(\operatorname{dist}_{P}\left(P_{i, 1}, P_{j, 2}\right)\right)$.

Statement (2) is to be interpreted as follows: for a given point $P_{i, 1}$ on frame 1, the best match among points $P_{j, 2}$ on frame 2 is chosen as the one that minimizes the "pointdistance" $\operatorname{dist}_{\mathrm{P}}\left(P_{i}, P_{j}\right)=\left[\left(x_{i}-x_{j}\right)^{2}+\left(y_{i}-y_{j}\right)^{2}\right]^{1 / 2}$, i.e., the 
standard Euclidean distance. Although such a general notation is not necessary at this stage, it will be useful in the remainder of the presentation as other distances are to be introduced.

To make the "minimum displacement" algorithm slightly more robust and avoid multiple matches to one and the same feature-point, a refinement is to set up the following optimization problem: find the global pairing $\{i(k), j(k)\}$ that minimizes the objective function

$\sum_{k=1}^{\min \left(n_{1}, n_{2}\right)} \operatorname{dist}_{P}\left(P_{i(k), 1}, P_{j(k), 2}\right)$

that is, a sum over all selected pairs of the distances between matched particles $P_{i(k), 1}$ and $P_{j(k), 2}$. This is a standard bijective graph optimization problem, which is difficult (and computationally expensive) to solve thoroughly for large numbers of points. An approximate solution is, however, easily found using the Vogel algorithm. The algorithm consists in considering for each particle image the best match and the second best match, then constructing a reasonable global optimum by picking particle pairs in the order of maximum difference between first and second best choices.

The simple match algorithm sketched above presents two shortcomings. First, from a computational point of view, it is unnecessarily expensive by requiring the tabulation of the distance between each feature-point of frame 1 and every feature-point of frame 2, however far apart from each other. It would thus be desirable to narrow down the match candidates using some suitable criterion. Secondly, a more serious problem is that the algorithm fails as soon as the interframe displacement of particles $\Delta r$ is not small with respect to the mean particle interdistance $\delta r$. In that case, nearest points from two successive images are likely to correspond to two different physical particles, and a "goodness-of-match" criterion more robust than the minimum displacement is required. In Capart (2000), it is shown that for the case of a rigidly-moving random dispersion of points, one must have $\Delta r / \delta r<0.35$ for minimum displacement matches to be reliable at the $90 \%$ confidence level. When the minimization improvement (3) is used, the condition relaxes to $\Delta r / \delta r<0.7$, still quite a stringent constraint in the case of rapidly moving, dense granular dispersions.

To address these problems, a first possibility is to use information from the particle's previous trajectory. Provided the past trajectory is known, a predictor step can be used to estimate the likely position of a particle on the next frame, and restrict the match candidates to those encountered in a limited search window around this position. Path regularity can then be used as an indicator of goodness-of-match (Sethi and Jain 1987; Jain et al. 1995). A refinement consists in solving a complex multi-frame optimization problem. Such methods, used for instance for the radar monitoring of air traffic (Brookner 1998), are successful for a number of PTV applications (Malik et al. 1993; Ushijima and Tanaka 1996) where particles are sparse and their trajectories well-behaved. However, when particle density is high and velocity fluctuations are important, these methods are highly unstable. This was experienced by the authors in their first attempts at imaging granular flows (Capart et al. 1997; Capart and Young 1998), with the consequence that close manual supervision was necessary to obtain reasonable results.

A second possibility is to use spatial rather than temporal information, and focus on pattern regularity rather than path regularity. Provided that particle neighbourhoods can be defined, these neighbourhoods can be used, on the one hand, to restrict the match candidates, and on the other hand, to extract point patterns that can be compared from one image to the next. These two operations can be respectively referred to as the "screening" and "selection" processes. Various ways of defining neighbourhoods have been developed (a review is given in Ahuja 1982) and applied to particle tracking applications. With reference to Fig. $4 \mathrm{a}-\mathrm{b}$, a common definition is to take a circular window of radius $R$ around feature-point $P_{i}$ of image 1 , and consider as match candidates the featurepoints of image 2 belonging to this window (or $R$-neighbourhood). A second circular window (possibly with a different radius $r$ ) can then be used to define a template of neighbours (or $r$-star) of particle image $P_{i}$, which can be compared with similar templates associated with the various match candidates on frame 2 (Fig. $4 \mathrm{a}-\mathrm{b}$ ). Such an approach has been used by Haynes and Turner (1992),
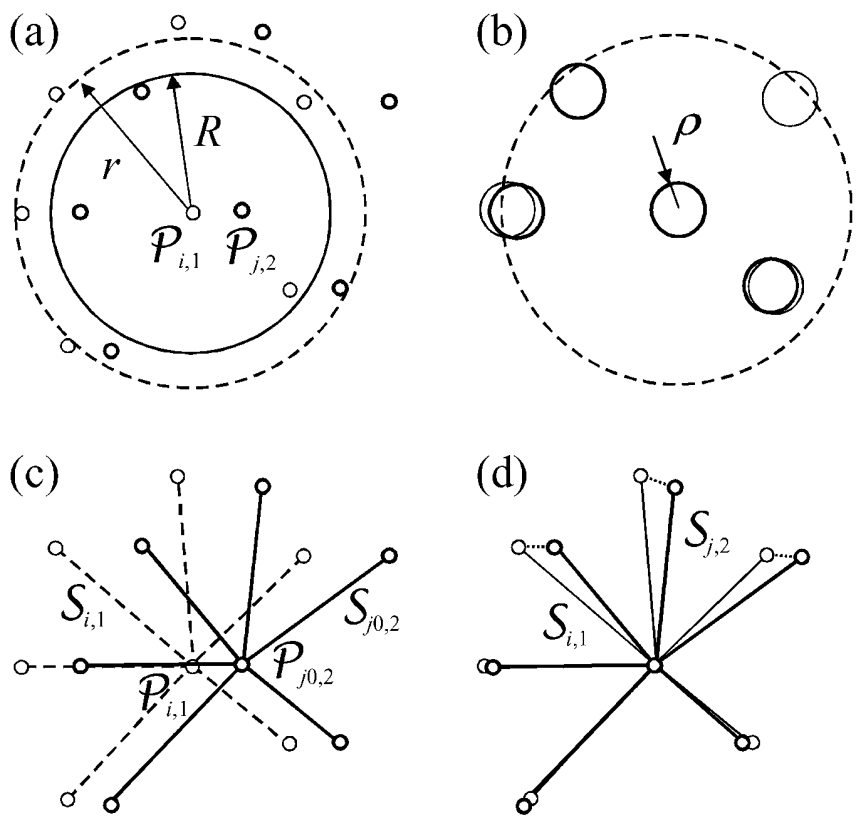

Fig. 4a-d. Screening (left) and selection (right) operations performed with circular windows (above) and Voronoï 1-stars (below): a a window of radius $R(-)$ is used to select the match candidates and a window of radius $r(--)$ defines the template to be matched; $\mathbf{b}$ goodness-of-match is evaluated by examining the area overlap between disks of radius $\rho$ centred on each featurepoint of the template; c the 1-star $S_{j 0,2}(-)$ of the minimumdisplacement match $P_{j 0,2}$ is used to select the match candidates, and the point's 1-star $S_{i, 1}(---)$ defines the match template; d goodness-of-match between stars $S_{i, 1}$ and $S_{j, 2}$ is estimated by looking at interpoint distances according to the Hausdorff-type measure of (5). Window-based algorithms (a) and (b) require the definition of three parameters $R, r, \rho$, whereas Voronoï methods (c) and (d) are non-parametric and are free to adapt to variations in the local state of the point dispersion 
Lloyd et al. (1992), and Ruan et al. (1999), choosing a particle area-overlap criterion for goodness-of-match between the templates (Fig. 4b). A similar approach could be devised with neighbourhoods (and stars) defined in terms of the $K(k)$ nearest neighbours (defining $K$-neighbourhoods and $k$-stars). However, both these approaches have the drawback that the radii or numbers of nearestneighbours considered must be defined a priori, and cannot adapt to variations or local gradients in point density (Ahuja 1982). The data structures necessary for constructing and handling the neighbour lists, which take the same form as the Verlet lists of molecular dynamics (Frenkel and Smit 1996), are also rather heavy to manipulate in comparison with a structure such as the Voronoï diagram.

Recently, Song et al. $(1996,1999)$ proposed to use the triangles of the Delaunay tesselation as match tokens. The present work proposes to resort to the Voronoï 1-star both to define a search neighbourhood and to provide a match template.

\section{3 \\ Voronoï match algorithm}

With reference to Fig. $4 \mathrm{c}-\mathrm{d}$, the proposed match algorithm involves the Voronoï construction for both screening and selection operations. It consists first in finding on frame 2 the minimum-displacement match $P_{j 0,2}$ corresponding to feature-point $P_{i, 1}$ of image 1 . The match candidates are selected as the feature-points on image 2 that belong to the Voronoï 1-star $S_{j 0,2}$ of point $P_{j 0,2}$. The Voronoï 1-star of each of the candidates is then compared with the 1-star of feature-point $P_{i, 1}$ to evaluate the goodness-of-match. Formally, the best match of point $P_{i, 1}$ is thus

$\operatorname{match}\left(P_{i, 1}\right)=\min _{P_{j, 2} \in S_{j 0,2}}\left(\operatorname{dist}_{S}\left(S_{i, 1}, S_{j, 2}\right)\right)$

where $S_{i}$ designates the Voronoï 1-star of point $P_{i}$, and $\operatorname{dist}_{S}\left(S_{i}, S_{j}\right)$ represents a suitably defined "star-distance" reflecting the degree of discrepancy between the patterns formed by two stars $S_{i}$ and $S_{j}$. For such a function, it is proposed to choose the median of the distances between the star extremities once the star centres have been made to coincide (Fig. 4d). Formally, this can be written

$\operatorname{dist}_{S}\left(S_{1}, S_{2}\right)=\operatorname{median}_{k_{1}=1 . . m_{1}}\left[\min _{k_{2}=1 . . m_{2}}\left|\left(\boldsymbol{r}_{k 1,1}-\boldsymbol{r}_{0,1}\right)-\left(\boldsymbol{r}_{k 2,2}-\boldsymbol{r}_{0,2}\right)\right|\right]$

where vertex $k_{1}=0$ (resp. $\left.k_{2}=0\right)$ is the centre of star $S_{1}$ (resp. $S_{2}$ ), vertices $k_{1}=1 . . m_{1}\left(\right.$ resp. $\left.k_{2}=1 . . m_{2}\right)$ are the extremities of star $S_{1}$ (resp. $S_{2}$ ), and $|r|=\left(x^{2}+y^{2}\right)^{1 / 2}$ is the usual Euclidean norm. Expression (5) features three successive stages: 1) a translation making the star centres coincide, allowing their shapes to be compared in a common frame of reference; 2) an inner loop based on the minimum norm, whereby for each extremity $\boldsymbol{r}_{k 1,1}$ of star $S_{1}$, the nearest extremity of star $S_{2}$ is found, and their distance from each other is added to a list; and 3 ) an outer loop based on the median norm, whereby the median value of the list is adopted as an overall measure of the discrepancy between the two stars $S_{1}$ and $S_{2}$. The above definition allows comparison between the shapes of any two stars, without requiring that they have the same number of branches. It corresponds to the Hausdorff distance (e.g., Goodrich et al. 1999), except for the use of the median rather than the maximum in the outer norm. The choice of the median is made in order to increase the robustness of the comparison: even the correctly matched stars (from a physical point of view) are expected to differ significantly at some of their extremities (when topological swap events result from flow deformation, when particle occlusion occurs, when particles reach the boundaries of the domain, etc.). Note that the star-distance defined above is "directed", i.e., in general, $\operatorname{dist}_{S}\left(S_{1}, S_{2}\right) \neq \operatorname{dist}_{S}\left(S_{2}, S_{1}\right)$. An undirected equivalent is easily defined as $1 / 2\left[\operatorname{dist}_{S}\left(S_{1}, S_{2}\right)+\operatorname{dist}_{S}\left(S_{2}, S_{1}\right)\right]$, but this was not found necessary in the present context.

Rather than simply setting $j(k)=\operatorname{match}(i(k))$, an optimization problem can again be set up to improve the matching and avoid multiple pairings with one and the same particle. This can be performed once again by seeking the global matching $\{i(k), j(k)\}$ that minimizes the objective function

$\sum_{k=1}^{\min \left(n_{1}, n_{2}\right)} \operatorname{dist}_{S}\left(S_{i(k), 1}, S_{j(k), 2}\right)$

where an arbitrarily large distance value is attributed to pairings involving match candidates that the screening process has not retained (i.e., such that $P_{j, 2} \notin S_{j 0,2}$ ). Approximate solutions to the optimization problem can again be obtained using the Vogel algorithm. It is shown in Capart (2000) that, for a rigidly moving random dispersion of points, screening the match candidates on the basis of their membership in the 1-star of the minimum displacement neighbour leads to a limitation in range of

$\Delta r / \delta r<1.5$ at the $90 \%$ confidence level. This is more than twice the range of the optimized minimum displacement algorithm, but may still be too restrictive in certain situations. In such cases, the Voronoï algorithm can be generalized to consider the Voronoï 2-star or higher-order stars of the feature-points. This was, however, found unnecessary in the applications examined in Sect. 3.

Qualitatively, the Voronoï match algorithm has the effect of pairing the neighbour feature-points that are characterized by similar Voronoï cells on successive images. For the granular flows examined, it has been verified that Voronoï cells tend to remain sufficiently stable along trajectories to allow the procedure to be successful. A close-up view of Voronoï diagrams corresponding to actual granular flow images illustrates this point in Fig. 5. More applications and results are detailed in Sect. 3.

\section{4}

\section{Natural-neighbour spatial filtering}

The algorithm above evidently does not succeed in matching all particle images correctly. It is thus desirable to have an automatic filtering procedure to remove mismatches from the set. In the next paragraph, a procedure for performing such a filtering based on multiple-image trajectories is presented. Relying on information derived from a single pair of image frames, it is also possible to devise spatial filtering procedures. Various proposals exist for this purpose. Most of them involve comparing each 


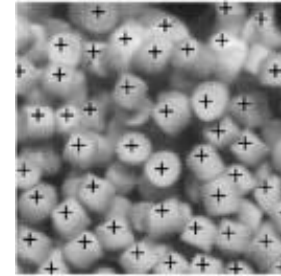

(a)

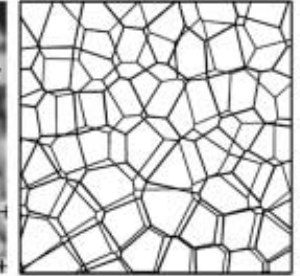

(b)

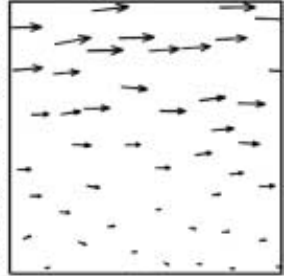

(c)
Fig. 5a-c. Overview of the Voronoï match algorithm: a image of a granular flow abstracted into point-like particles (+); b Voronoï diagrams constructed on these points (thin lines) and on the points of the next image of the sequence (thick lines); c displacement vectors (true scale) obtained by matching the Voronoï 1-stars. The result amounts to matching visually concordant Voronoï cells

velocity vector with a local average obtained from neighbouring feature-point velocities. An interesting alternative (Jähne 1995; Song et al. 1999) consists in exploiting flow invariants associated with polygonal shapes: an example is the area of Delaunay triangles constructed on passive particle tracers, which should be conserved in a divergence-free two-dimensional flow. In the present work, we are interested in the flow of a granular phase that is not locally divergence free (the granular dispersion is compressible), and we adopt the first approach. The construction of local averages, however, can again be based on the Voronoï construction.

The adopted approach resorts to the so-called "naturalneighbour weights", introduced by Sibson (1981) for interpolation purposes. The idea consists in subdividing each Voronoï tile $V_{i}$ into $m$ subtiles $W_{k}$, corresponding to the intersections of $V_{i}$ with the Voronoï polygons obtained if feature-point $P_{i}$ is removed from the set (Fig. 6). Weights can then be defined as

$\lambda_{k}=\frac{\operatorname{area}\left(W_{k}\right)}{\operatorname{area}\left(V_{i}\right)}$

These weights present various remarkable properties outlined in Sibson (1981). In particular, they can be used to derive $C^{2}$ interpolants (Sibson 1981), which have been used in a particle-tracking context by Lloyd et al. (1995). By construction, the weights $\lambda_{k}$ sum to 1 and reflect the proximity of point $P_{i}$ with each of the extremities of its associated 1-star (Fig. 6). It is thus possible to define the

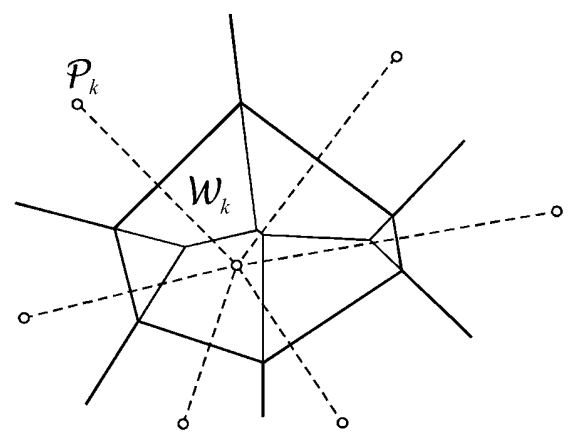

Fig. 6. Subtiling of the Voronoï cells used to define the Sibson weights "star-averaged" velocity associated with feature-point $P_{i}$ as the weighted sum

$\boldsymbol{v}_{i}^{*}=\sum_{k=1}^{m} \lambda_{k} \boldsymbol{v}_{k}$

where indices $k=1 . . m$ refer to the $m$ extremities of the 1-star of $P_{i}$, and the $\boldsymbol{v}_{k}$ are obtained from (1) once the matching has been performed. The above-defined average does not involve the velocity $\boldsymbol{v}_{i}$ at the star-centre, yet it presents the remarkable property of yielding $\boldsymbol{v}_{i}^{*}=\boldsymbol{v}_{i}$ if the velocities are governed by first-order variations (i.e., constant gradients) around feature-point $P_{i}$ (Sibson 1981). One can then define a second-order spatial difference operator as

$\Delta^{2} \boldsymbol{v}_{i}=\frac{\boldsymbol{v}_{i}^{*}-\boldsymbol{v}_{i}}{\operatorname{area}\left(V_{i}\right)}$

where $V_{i}$ is the Voronoï cell associated with point $i$. Expression (9) can be verified to reduce to the classical finite difference Laplacian if the local tesselation happens to be an orthogonal grid. A robust spatial filtering procedure can then be devised by imposing $\left|\Delta^{2} \boldsymbol{v}_{i}\right|<$ tol (where tol is a user-defined tolerance), and removing one by one the outliers by starting with those that create the maximum local "curvature". Implementation of the subtiling algorithm is complicated, but reasonably efficient (Sibson 1981). Once the subtiling is obtained, however, it can also be used for other purposes, for example to interpolate velocities onto a regular grid (as in Lloyd et al. 1995).

\section{5}

\section{Trajectory reconstruction and temporal filtering}

If a sequence of many images is recorded, displacement data obtained from each pair of frames according to the above algorithms can, of course, be concatenated and edited by using the more complete temporal information. Once successive match correspondences are known, pure concatenation of trajectory segments is trivial. Parallel to this operation, however, trajectories can be checked and edited using split-merge operations. The principles involved are similar to those of path-coherence based particle tracking (e.g. Ushijima and Tanaka 1996). The important difference is that these operations can now be performed on the basis of a pattern-based "skeleton" of trajectories rather than from scratch.

Temporal filtering can be performed efficiently using the 4-point stencils shown in Fig. 7 a. A subtrajectory $T_{i-1} . . T_{i+1}$ composed of points $P_{i-1} . . P_{i+2}$ located at positions $\boldsymbol{r}_{i-1} . . \boldsymbol{r}_{i+2}$ for successive times $t_{\mathrm{i}-1} . . t_{\mathrm{i}+2}$ is considered. Focusing on the central link $T_{i}$ of the subtrajectory, a robust local measure of path coherence is given by the "trajectory-distance"

$$
\begin{aligned}
\operatorname{dist}_{T}\left(P_{i}, P_{i+1}\right) & =\min \left(\Delta_{-}, \Delta_{+}\right) \\
& =\min \left(\left|\boldsymbol{r}_{i}^{-}-\boldsymbol{r}_{i}\right|,\left|\boldsymbol{r}_{i+1}^{+}-\boldsymbol{r}_{i+1}\right|\right)
\end{aligned}
$$

where $\boldsymbol{r}_{i}^{-}=2 \boldsymbol{r}_{i+1}-\boldsymbol{r}_{i+2}$ and $\boldsymbol{r}_{i+1}^{+}=2 \boldsymbol{r}_{i}-\boldsymbol{r}_{i-1}$ are respectively backward and forward extrapolations based on the surrounding segments. One can again filter out likely mismatches by imposing $\operatorname{dist}_{T}\left(P_{i}, P_{i+1}\right)<$ tol. The motive for using the minimum norm is illustrated in Fig. 7b: to avoid 

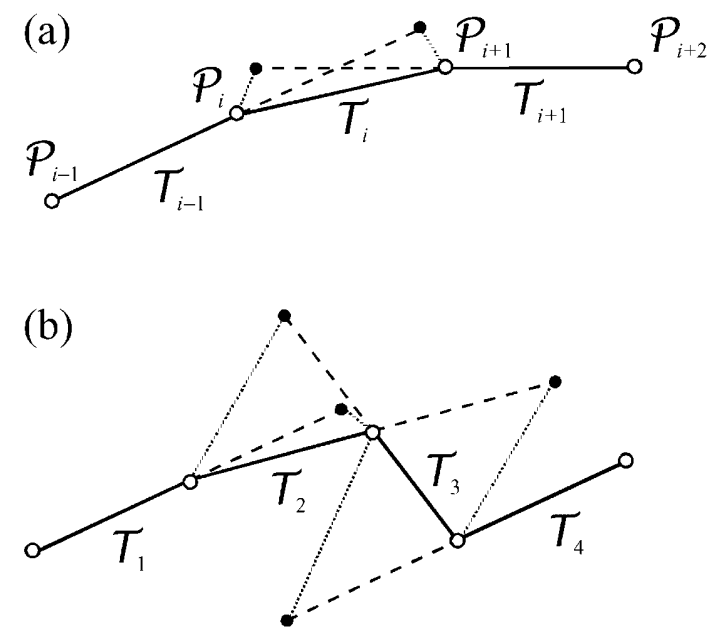

Fig. 7a-b. Trajectory filtering: a four-point stencil used to evaluate the local path coherence; $\mathbf{b}$ by resorting to the minimum norm in (10), link $T_{3}$ can be discarded without discarding link $T_{2}$

discarding correct links, only those presenting excess discrepancies with respect to both the forward and backward extrapolations are filtered out.

Once trajectories are "split" at their weak links, one can attempt "merge" operations for trajectory ends that appear to fit together well according to the same criterion. An elegant way to perform the "split" and "merge" operations in one single sweep consists in recasting the problem as one of finding for each pair of frames the global match $\{i(k), j(k)\}$ that minimizes the objective function

$$
\sum_{k=1}^{\min \left(n_{1}, n_{2}\right)} \operatorname{dist}_{T}\left(P_{i(k), 1}, P_{j(k), 2}\right)
$$

and keeping only the matches that satisfy a given tolerance. By starting from the "trajectory skeleton" obtained by the Voronoï matching process, trajectory-based improvements can be made in substeps that do not require the handling of more than two frames at a time. In this way, one avoids the algorithmic complexity involved in keeping track of various multiframe trajectories.

In a granular flow context, trajectory information can be quite useful, for instance, to visualize the granular motions or to extract autocorrelation statistics. These are of theoretical importance in granular flows, for instance, to estimate collisional frequencies or compare self-diffusion statistics with those of computational simulations.

\section{6}

\section{Granular concentration estimation}

Because they tile the plane without gaps, the Voronoï cells and Delaunay triangles can be used to provide a local measure of the planar density $\eta$ of feature-points observed in an image. One only needs to take the reciprocal of the areas, which corresponds to 1 particle in the case of a Voronoï cell, and 1/2 particle in the case of a Delaunay triangle. When dealing with two-dimensional granular dispersions (for instance, disks or spheres constrained to move between two closely spaced walls), this constitutes an immediate estimate of concentration. In contrast, when three-dimensional dispersions of grains are imaged through a sidewall, the estimation of volumetric concentration $\phi$ from two-dimensional images is a nontrivial task. Various authors (Louge and Jenkins 1997; Kanda et al. 1999) propose to use luminance information for this purpose. Even in laboratory conditions, however, illumination conditions are difficult to control precisely. In the present work, it is therefore sought to use exclusively the information associated with particle positions, which is much less dependent on lighting.

Situations are different for the dilute and dense cases. In the dilute limit, for instance, in the case of sparse dispersions of particles restricted to a thin sheet (e.g., a laser light sheet), one can relate the $3 \mathrm{D}$ concentration to the $2 \mathrm{D}$ density of visible particles by assuming that their positions obey a Poisson process (Adrian 1991). A short analysis sketched in Appendix 2 yields:

$\eta=\frac{4}{\pi d^{2}}\left[1-\exp \left(-\frac{3}{2} \frac{\phi \Delta z}{d}\right)\right]$

where $\eta$ is the number of visible particle centroids by unit image surface, $\phi$ is the volumetric solid concentration, and where it was assumed that spherical particles of diameter $d$ are contained in a viewing volume of thickness $\Delta z$. From (12) it is immediately apparent that as soon as the ratio $\phi \Delta z / d$ grows, occlusion effects cause the surface density $\eta$ to lose its sensitivity to changes in $\phi$.

Fortunately, when the dispersion becomes dense, excluded volume effects intervene, and the particle positions are no longer governed by a random Poisson process. When the mean particle interdistance becomes of the order of the diameter, neighbouring particles are forced to organize with respect to each other in a type of glassy state (Allen and Thomas 1999). This creates shortrange correlations between grain positions and opens a possibility of trying to link solid concentration with local descriptors of particle configurations. Various approaches can be used to construct such descriptors (for a review and comparison, see Wallet and Dussert 1998). The Voronoï diagram provides once again a possible tool, assessed by Wallet and Dussert (1998) to be one of the best from the point of view of discrimination power and stability (the best results for their tests were, however, obtained with minimal spanning tree approaches). In the present work, three Voronoï-based indicators are tested.

The first is an estimator for the point density $\eta$ :

$\eta_{i}=\frac{1}{\operatorname{area}\left(V_{i}\right)}$

where $V_{i}$ is the Voronoï cell enclosing point $i$. The second is the "roundness factor", which provides a descriptor of the shape of Voronoï polygons:

$\xi_{i}=\frac{4 \pi \operatorname{area}\left(V_{i}\right)}{\left[\operatorname{perimeter}\left(V_{i}\right)\right]^{2}}$

Finally, a third indicator reflects the local Voronoï "area disorder" and is defined as:

$\chi_{i}=\frac{1}{1+\sigma / \mu}$ 
where

$\sigma^{2}=\sum_{k=1}^{m} \lambda_{k}\left[\operatorname{area}\left(V_{k}+W_{k}\right)-\mu\right]^{2}$

and

$\mu=\sum_{k=1}^{m} \lambda_{k} \operatorname{area}\left(V_{k}+W_{k}\right)$

where indices $k=1 . . m$ designate the $m$ natural neighbours of point $i$, and where the weights $\lambda_{k}$ and polygons $V_{k}$ and $W_{k}$ are defined as in (7) (see also Fig. 6). The "area disorder" estimator can be interpreted as the normalized variance of the areas of the "petals" of the Voronoï "flower" shown in Fig. 8. The estimator is a local version of the global area disorder defined by Marcelpoil and Usson (1992). The weighted averages are chosen in analogy with the treatment of mixture densities in Duda and Hart (1973). Note that in contrast with (13), estimators (14) and (15) are dimensionless and scale-invariant.

For the three indicators above, relationships with concentration $\phi$ are sought in the following power-law form:

$$
\begin{aligned}
\frac{\phi}{\phi_{\mathrm{rcp}}} & =\left(\frac{\eta}{\eta_{\mathrm{rcp}}}\right)^{\alpha}=\eta^{\prime^{\alpha}} \\
\frac{\phi}{\phi_{\mathrm{rcp}}} & =\left(\frac{\xi-\xi_{0}}{\xi_{\mathrm{rcp}}-\xi_{0}}\right)^{\beta}=\xi^{\beta} \\
\frac{\phi}{\phi_{\mathrm{rcp}}} & =\left(\frac{\chi-\chi_{0}}{\chi_{\mathrm{rcp}}-\chi_{0}}\right)^{\gamma}=\chi^{\prime \prime}
\end{aligned}
$$

where indices " $r c p$ " and " 0 " designate the state of random close packing and the dilute state, respectively; $\phi_{\text {rcp }} \approx 0.64$ for spheres (Allen and Thomas 1999); $\xi_{0} \approx 0.72$ and $\chi_{0} \approx 0.80$ are obtained from Monte-Carlo simulations (as they concern the dilute state, the values do not vary with particle shape); random close-packing values $\eta_{\text {rcp }}$, $\xi_{\mathrm{rcp}}$, and $\chi_{\mathrm{rcp}}$, as well as exponents $\alpha, \beta$, and $\gamma$ remain to be determined from calibration tests.

\section{3}

\section{Applications and results}

The algorithms above were tested on a number of liquidgranular flow experiments, which also motivated and

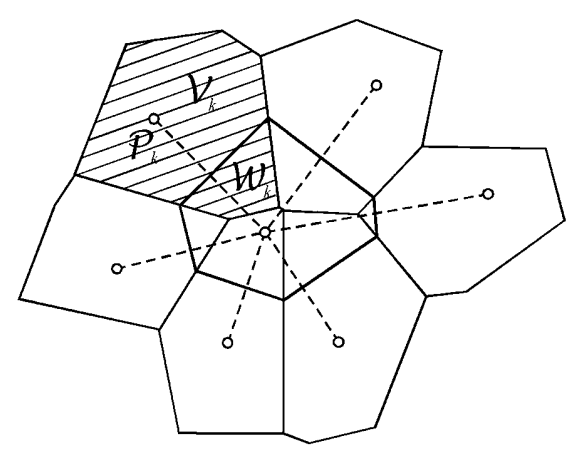

Fig. 8. The union of a neighbour cell $V_{k}$ with subtile $W_{k}$ forms a "petal" of the Voronoï "flower". The variance of the petal areas can be used to construct a local "area disorder" estimator guided the developments. In the present report, three applications are selected as testing grounds for the algorithms: (i) homogeneous fluidization tests; (ii) steady uniform debris flow; (iii) transient debris surges. These contrasted applications make it possible to highlight different features of the proposed methods.

\section{1}

\section{Homogeneous fluidization}

In order to test the concentration estimators, it is desirable to obtain states of homogeneous dispersion for various values of the solid fraction. A most convenient way of achieving this is to set up fluidization cell experiments. These consist of subjecting a static array of grains to an upward fluid flux, causing the granular assembly to expand until the voidage is such that the interphase drag balances the submerged weight of the granular phase. At some stage, when concentrations become lower than the random loose-packing concentration $\phi_{\text {rlp }} \approx 0.55$, the grains become mobile with respect to each other and undergo disordered fluctuating motions. Provided one can avoid the regions of instabilities (Batchelor 1988), a statistically homogenous state is obtained in which the grains randomly explore a variety of local configurations. Such conditions are the ideal ones to test and calibrate patternbased concentration estimators.

In the present work, tests were performed with light spherical grains fluidized by a water current. The grains (artificial pearls) have a diameter $d=6.1 \mathrm{~mm}$ and a relative density $\rho / \rho_{\mathrm{w}}=1.048$, where $\rho$ is the density of the granular material and $\rho_{\mathrm{w}}$ is the density of water. The cylindrical fluidization cell has a height of $25 \mathrm{~cm}$ and a diameter of $10 \mathrm{~cm}$. The lower part of the cell is filled with a 5 -cm-deep layer of small lead spheres in order to diffuse the incoming water current. Finally, the cylinder is fitted with a plane rectangular observation window of dimensions 5 by $10 \mathrm{~cm}$ allowing one to film the dispersion without optical distortion. Varying the upward water velocity in a range of 1 to $3 \mathrm{~cm} / \mathrm{s}$ enables observations of fluidized granular dispersions having concentrations between $\phi=0.2$ and $\phi=0.55$. The relationship between concentration and fluidizing flux was verified to correspond reasonably well with the Richardson-Zaki empirical relation (Richardson and Zaki 1954). As the granular motions are rather slow in this case, tracking of the particles constitutes a simple matter, and was carried out only to verify that the state of the dispersion was close to statistically homogenous. The measured kinetic energy of the velocity fluctuations was found to agree well with the relationship proposed by Batchelor (1988) for homogeneous fluidization conditions. These verification data are presented in Capart (2000).

The main purpose of the fluidization experiments is to test the pattern-based concentration indicators. Once particle centroids are located with the algorithms of Appendix 1, the Voronoï diagram can be constructed on these feature-points, and estimators (13)-(15) obtained for each of the Voronoï cells. To prevent edge effects from biasing the statistics, the criterion of Kenkel et al. (1989; see Okabe et al. 1992) is used to discard Voronoï cells close to the image boundaries. It consists in excluding 
from consideration all cells for which a circle centred at any vertex and passing through the cell feature-point and two of its natural neighbours intersects the boundary (Fig. 9). In Fig. 10, the normalized indicators (18) are plotted against the normalized concentration. Each data point represents an average over 20 frames and over all the visible particles (about 100 particles per image). Power-law fits are seen to well approximate the data trends. The calibrated constants take values $\eta_{\mathrm{rcp}}=1.40 / d^{2}$ (where $d$ is the particle diameter); $\xi_{\text {rcp }}=0.84 ; \chi_{\text {rcp }}=0.92 ; \alpha=6.0$; $\beta=\gamma=3.5$. The particle number indicator (13) is seen to present the least scatter, but the sensitivity is rather low. In fact, the sensitivity to particle number is much lower than was anticipated by Capart et al. (1997) on the basis of a geometrical reasoning. The two pattern-based indicators (14) and (15), on the other hand, present a higher degree of scatter, but with better sensitivity. Overall, the results

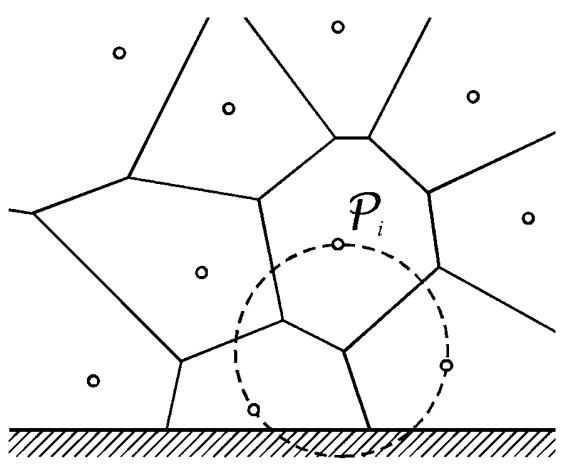

Fig. 9. Criterion of Kenkel et al. (1989) for the identification of Voronoï cells subject to boundary effects (after Okabe et al. 1992). Because the circle (- - ) intersects the boundary, featurepoint $P_{i}$ (and its associated templates) is excluded from consideration

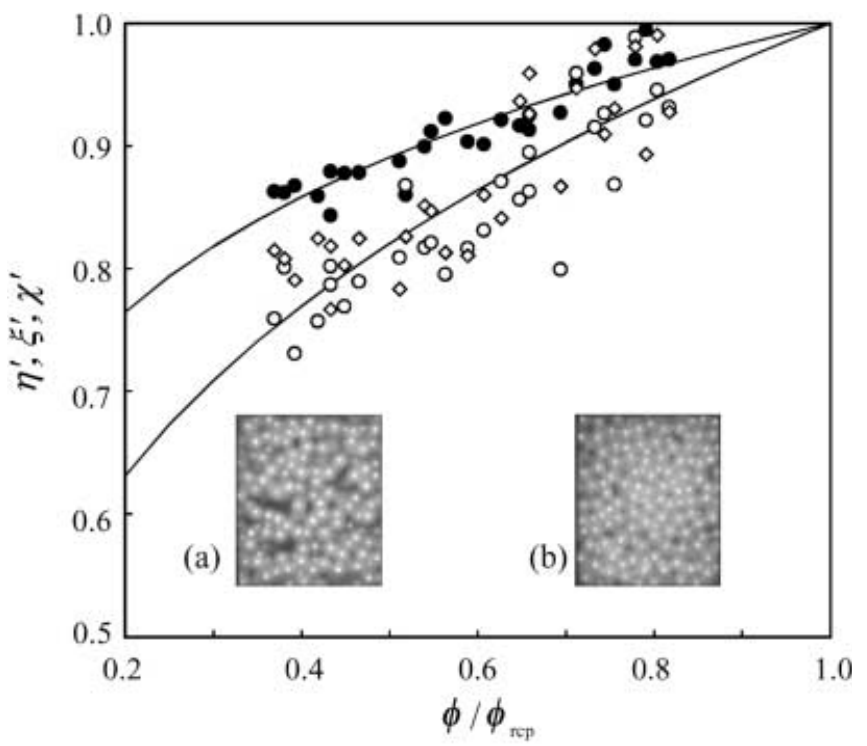

Fig. 10a, b. Relationship between the volumetric grain concentrations and the Voronoï-based estimators: $(\bullet)$ point density $\eta$ '; $(\bigcirc)$ roundness $\xi^{\prime} ;(\diamond)$ area disorder $\chi^{\prime} ;(-)$ power law fits (the roundness and area disorder estimators are approximately fitted by a single curve). Inset: images of the dispersions for a $\phi \approx 0.3 ; \mathbf{b} \phi \approx 0.5$ suggest that the indicators can be used to measure solid concentrations, under the conditions that (i) averages can be performed over a large amount of individual data points; (ii) the granular dispersion behaves locally in a random fashion close to the conditions of the fluidization tests; (iii) calibration can be performed for a given material in lighting conditions similar to those of the measurements. One case where these conditions are met is presented in Sect. 3.2.

\section{2}

\section{Steady uniform debris flow}

To investigate the vertical structure of free-surface liquidgranular flows, it is of particular interest to be able to materialize steady uniform flow conditions. A recirculating flume of novel design was developed for this purpose by scientists of the Università degli Studi di Trento, Italy (Armanini et al. 2000). With reference to Fig. 11, the flume is composed of two main components: a tilting glass-walled channel, $6 \mathrm{~m}$ in length and narrowed to $20 \mathrm{~cm}$ in width, in which free-surface flows are observed; a conveyer belt, connected to the channel by chute guides, which recirculates both water and sediment. During a run, except for minor losses, the set-up forms a closed loop in which a determined volume of water and granular material circulates. The global flow in the channel is governed by the volumes of water and sediment and by the channel angle, which can be varied from 0 to 25 degrees. The apparatus is used to study steady uniform flows of various degrees of maturity in rigid-bed and loose-bed conditions (Armanini et al. 2000).

To test the Voronoï imaging methods, one of the runs analysed by Capart et al. (2000) is examined here in detail. It consists of a rapid high-concentration debris flow in contact with the rigid flume bottom. The sediment material is composed of roughly identical cylinder-shaped PVC granules having the following characteristics: diameter $=3.2 \mathrm{~mm}$; height $=2.8 \mathrm{~mm}$ (hence an equivalent spherical diameter $d=3.5 \mathrm{~mm}$ ); relative density $\rho / \rho_{w}=1.540$. The flow conditions were the following: slope $=3$ degrees; total discharge (water + sediment $)=12.4 \mathrm{l} / \mathrm{s}$; delivered sediment concentration (sampled at the outlet of the flume) $\bar{\phi}=0.49$. The image sequence was acquired by filming the flow through the flume sidewall, using a CCD camera with an image resolution of $320 \times 280$ pixels at a frame rate of $500 \mathrm{~Hz}$.

Figure 12 presents typical particle tracking results obtained using the Voronoï methods. One original image of the sequence is shown in Fig. 12a. The flow is about $3 \mathrm{~cm}$ in depth and is heavily loaded with PVC particles. Voronoï matching results for two subsequent frames are shown in Fig. 12b. To improve the matching results, one additional criterion was imposed for the screening of match candidates, and consists in an elliptic window constraining the allowable interframe displacements (Fig. 13). With reference to Fig. 13, the following ellipse parameters were adopted for the run under consideration: $u_{0}=2 \mathrm{~mm} ; v_{0}=0 ; w_{1}=3 \mathrm{~mm} ; w_{2}=2 \mathrm{~mm}$. The constraint is applied uniformly to the whole field, and exploits information known a priori about the flow to reduce the number of candidates with respect to the more general Voronoï 1-star screening criterion. For the selection 

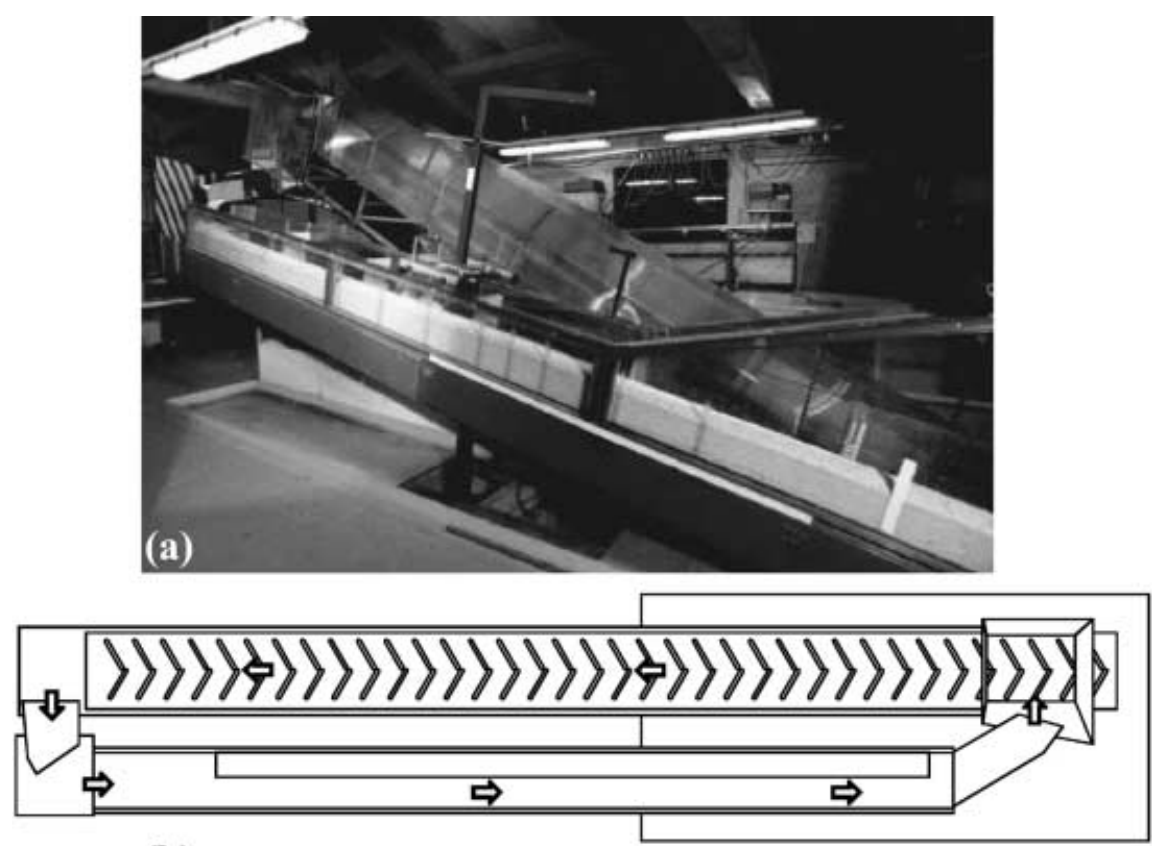

(b)
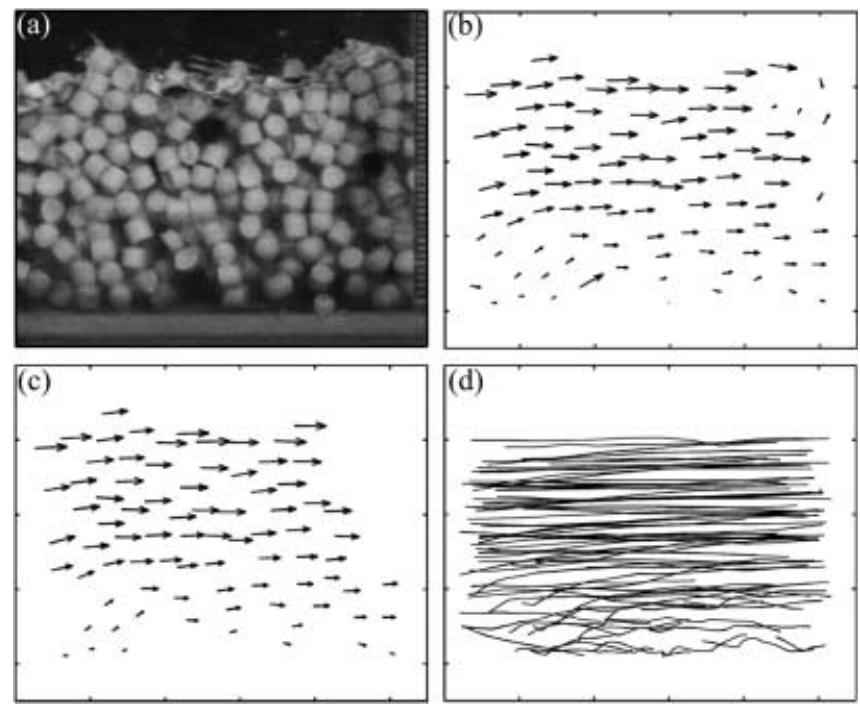

Fig. 12a-d. Analysis of steady uniform debris flow experiments: a original image frame; $\mathbf{b}$ displacement field derived from two successive frames (displacement vectors are shown true scale); c displacement field after application of natural-neighbour spatial filtering; d grain trajectories reconstructed from 20 successive frames (with temporal filtering). Tick marks are placed on the axes with a spacing of $1 \mathrm{~cm}$

operation, the Voronoï algorithm is used without changes. In Fig. 12b, some displacements are seen to be likely mismatches. Fig. 12c shows the displacement field after the application of the natural-neighbour spatial filtering procedure of Sect. 2.4. The procedure is successful at removing the most irregular displacement vectors, without pruning too many correct ones. Finally, Fig. 12d shows trajectories reconstructed from 20 successive frames, after application of the temporal "split and merge" filtering of Sect. 2.5.
Fig. 11a, b. Recirculating flume developed at the Università degli Studi di Trento (Armanini et al. 2000) for the experimental study of steady uniform debris flows: a side view; b plane view (photograph courtesy of L. Guarino)

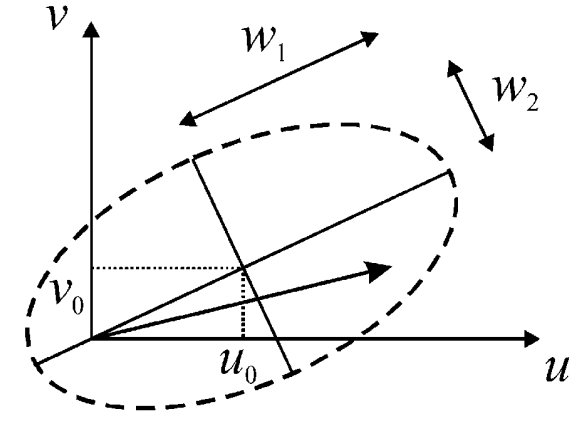

Fig. 13. Elliptical window used to refine the screening of the match candidates. The ellipse constrains the acceptable interframe displacements

Granular trajectories offer a vivid view of the flow behaviour. The lowermost part of Fig. 12d exhibits irregular trajectories characteristic of saltating motions (Lee and Hsu 1994) interrupted by collisions with the rough bed and with other grains. Trajectories in the upper part, on the other hand, present smaller fluctuations. These features are characteristic of the rapid (collisional) granular flow regime (Campbell 1990). Such dense trajectories, obtained by tracking all visible grains in an intensely sheared fluctuating ensemble, have not been obtained before from experiments. They demonstrate both the challenging features of granular flows and the robustness of the Voronoï methods.

The Voronoï methods function in a fully automated way. It is thus possible to apply the steps demonstrated in Fig. 12 to a full sequence of images. This is necessary if one seeks to derive accurate statistics for vertical flow profiles. From numerical simulations, Louge and Jenkins (1997) found that a sample size of a minimum of 10,000 measurements was necessary for this purpose, which is far beyond what can be achieved using manual or even semi- 
automated procedures. For the flow under consideration, a sequence of 500 frames was analysed, producing around 50,000 individual measurements for velocity and concentration. The good performance of the Voronoï procedure is reflected by the vector yields obtained in this case: on average, 104 particles are identified on each frame, of which 94 are matched to particles on the next frame, with 92 acceptable velocity vectors remaining after temporal filtering. The percentage of successful matches is therefore around $92 / 104=88 \%$.

The resulting averaged vertical profiles, obtained by subdividing the flow into 20 horizontal layers, are presented in Fig. 14. Profiles are shown for the mean longitudinal velocity $\langle u\rangle$, for the mean concentration $\phi$ derived from averages of the point density, roundness, and area disorder indicators $\langle\eta\rangle\langle\xi\rangle$ and $\langle\chi\rangle$ and for the granular temperature $\theta$. The latter is defined in analogy with the kinetic theory of gases and constitutes a measure of the strength of the particle velocity fluctuations (Jenkins and Hanes 1998). It is estimated here as

$\theta=\frac{1}{3}\left\langle\boldsymbol{v}^{\prime} \cdot \boldsymbol{v}^{\prime}\right\rangle \approx \frac{1}{2}\left(\left\langle u^{\prime 2}\right\rangle+\left\langle v^{\prime 2}\right\rangle\right)$

where $\boldsymbol{v}^{\prime}=\boldsymbol{v}-\langle\boldsymbol{v}\rangle$ is the fluctuation velocity vector, and $u^{\prime}=u-\langle u\rangle$ and $v^{\prime}=v-\langle v\rangle$ are its components along directions parallel and normal to the bed, respectively. The brackets $\langle\cdot\rangle$ denote an average performed over time and over all particles located within each horizontal layer. The estimated root-mean-square (rms) error of particle position is of the order of 0.25 pixel, or $2 \%$ of the diameter of the particles. At mid-depth of the flow illustrated in Fig. 14, this leads to an expected rms error of $\varepsilon=0.05 \mathrm{~m} / \mathrm{s}$ for the individual velocity measurements. The relative errors in individual measurements of velocity $v$ and granular temperature $\theta$ can then be estimated as:

$e_{v}=\frac{\varepsilon}{\langle u\rangle}=3 \% ; \quad e_{\theta}=\frac{\varepsilon^{2}}{\left\langle\boldsymbol{v}^{\prime} \cdot \boldsymbol{v}^{\prime}\right\rangle}=20 \%$.

Thus a small relative rms error is obtained for the velocities, while a greater but still reasonable relative error affects the granular temperatures. Errors in velocities cancel out when the mean velocity profile $\langle u\rangle$ is estimated from the large set of measurements. By contrast, errors in the granular temperature $\theta$ do not average out upon aggregation of a large measurement sample. Thus even small errors in particle position (only $2 \%$ of the particle diameter) are enough to significantly perturb granular temperature measurements. Because error noise is added in, the profile of Fig. $14 \mathrm{c}$ is likely to overestimate by about $20 \%$ the actual strength of the velocity fluctuations. More information about PTV errors is given in Veber et al. (1997).

In the collisional regime, the randomly fluctuating dispersion of grains is expected to be in a state similar to the fluidized state of Sect. 3.1. Hence the concentration indicators tested in the fluidization experiments should respond correctly to changes in the volumetric concentration. This is not the case for all granular shear flows. For the slower and denser frictional flow regime, in particular, the local arrangement of grains can be governed by a flow-dependent microstructure, leading to a breakdown of the pattern-based estimates. In such cases, it is probably necessary to resort to other measurement principles (stereo imaging, radiometric density meters, or other techniques).

Since the PVC granules are cylinder-shaped and respond differently to illumination, some of the calibration parameters involved in the concentration relations had to be obtained anew. A value for the random close-packing concentration $\phi_{\mathrm{rcp}}=0.69$ was obtained by measuring the concentration of a static, close-packed assembly of the PVC grains. Values for parameters $\eta_{\mathrm{rcp}}, \xi_{\mathrm{rcp}}$, and $\chi_{\mathrm{rcp}}$ were further recalibrated by comparing sidewall and outlet measurements of the delivered concentration $\bar{\phi}$, defined as the ratio of depth-integrated granular discharge over the total depth-integrated discharge. Calibration was performed by requiring that the sidewall integrated $\bar{\phi}$ be equal to the bulk $\bar{\phi}$ sampled by trapping outgoing volumes of water and grains at the flume outlet, i.e.,

$\bar{\phi}=\frac{\int_{y_{0}}^{y_{0}+h} \phi\langle u\rangle \mathrm{d} y}{\int_{y_{0}}^{y_{0}+h}\langle u\rangle \mathrm{d} y}=\left.\frac{\text { volume of grains }}{\text { total volume }}\right|_{\text {outlet }}$,

in which $y_{0}$ is the elevation of the rigid bed and $h$ is the flow depth. The values obtained by this procedure are $\eta_{\mathrm{rcp}}=0.60 / d^{2} ; \xi_{\mathrm{rcp}}=0.835$ and $\chi_{\mathrm{rcp}}=0.915$. All other parameters in (18) are left unchanged. With these recalibrated values, the three concentration profiles in Fig. 16b are seen to be in approximate agreement with each other. Recalibrated values of $\xi_{\text {rcp }}$ and $\chi_{\text {rcp }}$ are rather close to the values derived from the fluidization tests, whereas $\eta_{\mathrm{rcp}}$ changes drastically. Thus the pattern-based indicators (roundness and area disorder) appear to present a reasonable degree of stability with respect to particle shape and illumination. In contrast, the point-density estimator $\eta$ is found to be highly shape- and/or illuminationdependent. Its use is therefore not recommended unless one can perform well-controlled calibration tests in the same conditions as the experiments of interest.

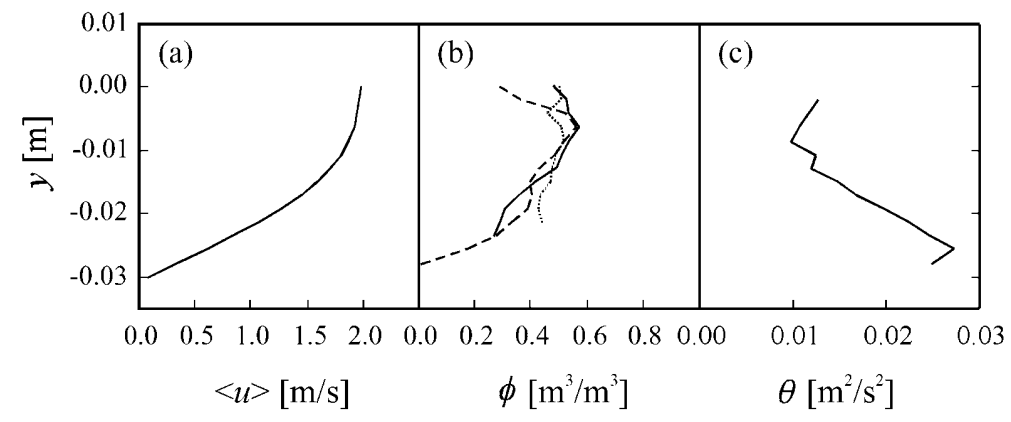

Fig. 14a-c. Averaged vertical profiles for the steady uniform debris flow experiments: a longitudinal velocity $\langle u\rangle$; b granular concentration $\phi$ as derived from (-) point density, (- - -) roundness, and ( $\cdots)$ area disorder indicators; $\mathbf{c}$ granular temperature $\theta$ 
The profiles of Fig. 14 further highlight the characteristics of collisional flow. They are similar to profiles obtained for rigid bed conditions in computational simulations for dry, two-dimensional dispersions of disks (see the illustrative example in Campbell 1990), even though we are dealing here with a three-dimensional dispersion of particles embedded in water. Thus the description of Campbell (1990) applies here with few changes: the mean velocity presents its steepest gradient at the bottom; concentration is low near the bottom, reaches a maximum towards the centre, and tails off again near the free surface; the granular temperature is large near the bottom (where a large temperature generation results from the steep velocity gradient) and decreases towards the free surface. The quantitative information gathered from these profiles complements the qualitative picture provided by the trajectories of Fig. 12d: a dense upper layer of grains flows rapidly over a relatively dilute layer of highly fluctuating grains close to the bottom, which supports the submerged weight of the upper layer through collisional contacts. For more details concerning the physical analysis of such measurements, the reader is referred to Guarino (1998), Armanini et al. (2000), and Capart et al. (2000).

\section{3}

\section{Dam-break-induced debris waves}

Transient liquid-granular flows constitute a challenging application for experimental techniques. Such flows are of importance in geophysical fluid mechanics, for instance, in mountain catchments where debris flows usually take the form of highly unsteady debris surges (Takahashi 1991). Dam-break waves constitute one of the possible triggering mechanisms for debris flows (Costa and Schuster 1988; Capart et al. 2001), and are relatively simple to set up in idealized laboratory conditions (Capart and Young 1998). It is this problem which first motivated the development of the present Voronoï imaging methods.

The flow configuration considered is shown in Fig. 15. In this small-scale laboratory set-up, clear water is retained behind a sluice gate (representing an idealized "dam") in a $10 \mathrm{~cm}$ wide rectangular channel. A sudden channel enlargement occurs $20 \mathrm{~cm}$ downstream of the gate, where the flume width goes abruptly from $10 \mathrm{~cm}$ to $20 \mathrm{~cm}$. A hori-

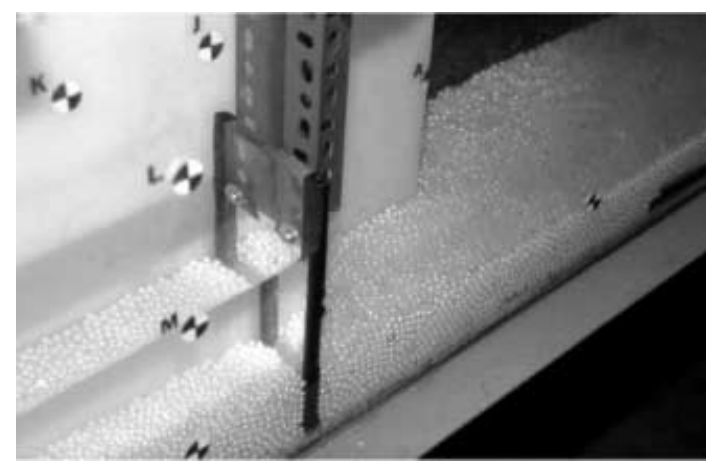

Fig. 15. Dam-break wave over granular bed experimental set-up installed at the Hydrotech Research Institute of the National Taiwan University. Shown are the initial conditions before removal of the sluice gate zontal layer of loose granular material, $5 \mathrm{~cm}$ in depth, fills the channel bottom both upstream and downstream. Upstream of the gate, the water stage rises to a depth of $10 \mathrm{~cm}$ above the horizontal granular bed, whereas the downstream water stage coincides with the bed surface. The granular phase is thus saturated with water throughout the domain. The granular material is composed, as in Sect. 3.1, of artificial pearls having a diameter $d=6.1 \mathrm{~mm}$ and a relative density $\rho / \rho_{w}=1.048$. A dam-break flow analogue is obtained by impulsively raising the sluice gate (within $50 \mathrm{~ms}$ ), releasing a water wave that entrains granular material from the loose bed. The flow is filmed from the side and from above using a CCD camera operating at a resolution of $256 \times 256$ pixels and frequencies of $100 \mathrm{~Hz}$ (side view) and $125 \mathrm{~Hz}$ (top view). Lighting is provided by two $500 \mathrm{~W}$ projectors oriented at angles of $45^{\circ}$ with respect to the camera axis. More details about the experimental procedure are provided in Capart and Young (1998).

We first focus on the initial stages of the flow, before the wave reaches the channel enlargement, which correspond to the prismatic channel conditions treated in Capart and Young (1998). For these initial stages, the flow is essentially two-dimensional (it is uniform across the width), and can be entirely characterized by sidewall observations. In Capart and Young (1998), granular velocity fields for this flow were presented at selected times, and were obtained through a painstaking manually supervised procedure. The Voronoï methods now allow the tracking to be performed automatically, with the same accuracy and much less effort. In particular, tracking can be performed over many more images in order to reconstitute the long time trajectories of the grains. This is shown in Fig. 16. Figure 16a presents an original image frame taken at a time of $0.17 \mathrm{~s}$ after the dam-break. Figure 16b shows the corresponding displacement field derived from the last two images, whereas Fig. $16 \mathrm{c}$ presents the same data after natural-neighbour spatial filtering. The results of Fig. 16c
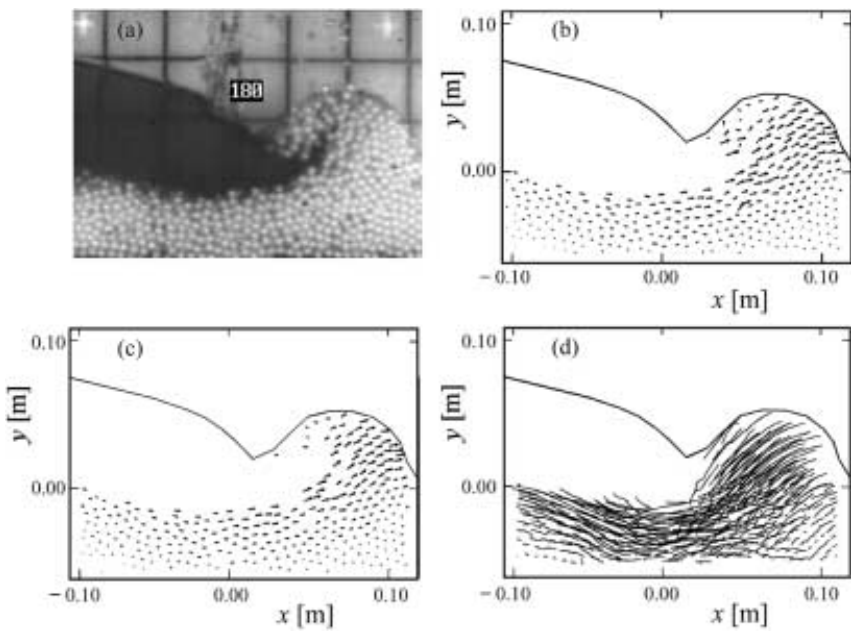

Fig. 16a-d. Side-view analysis for the dam-break experiments: a original image frame at time $t=0.17 \mathrm{~s}$; b displacement field derived from two successive frames (displacement vectors are shown true scale); c displacement field after application of natural-neighbour spatial filtering; $\mathbf{d}$ grain trajectories reconstructed from 18 successive frames (with temporal filtering) 
compare quite favourably with the manually obtained fields of Capart and Young (1998). Finally, Fig. 16d is a plot of the granular trajectories over the first 18 frames of the sequence following the dam-break. The flow field is characterized by rapid deformation and complex trajectories are obtained. Downstream particles surge upwards with the wave, and are replaced by other particles flowing in from upstream (hence their trajectories cross in the course of the transient flow). This new plot would have been very difficult to obtain without robust, fully automated procedures.

Beyond the enlargement, the two-dimensional debris wave expands into a three dimensional surge overrunning the granular bed. The wave can then be followed by filming from above, and tracking the visible grains of the flow free surface. This is shown in Fig. 17, where reconstructed granular trajectories are plotted for selected instants. The surge head is characterized by a sharp wavefront, or "erosional bore", which radiates from the enlargement and impulsively sets in motion a layer of bed material. The wavefront evolves from the debris snout shown in crosssection on Fig. 16. Seen from above, it creates a zone of sharp velocity gradients that the Voronoï methods are seen to handle rather well. For physical descriptions and numerical simulations of such debris surges, the reader is referred to Capart and Young (1998) and Capart et al. (2001).

\section{4}

\section{Conclusions}

In the present work, the properties of the Voronoï diagram have been exploited to derive a family of methods for the imaging analysis of particulate flows. The methods include a novel matching algorithm for particle tracking velocimetry and a set of indicators for the three-dimensional granular concentration. They also comprise procedures for spatial and temporal filtering, based respectively on pattern and path coherence. The techniques have been successfully tested on various liquid-granular flow
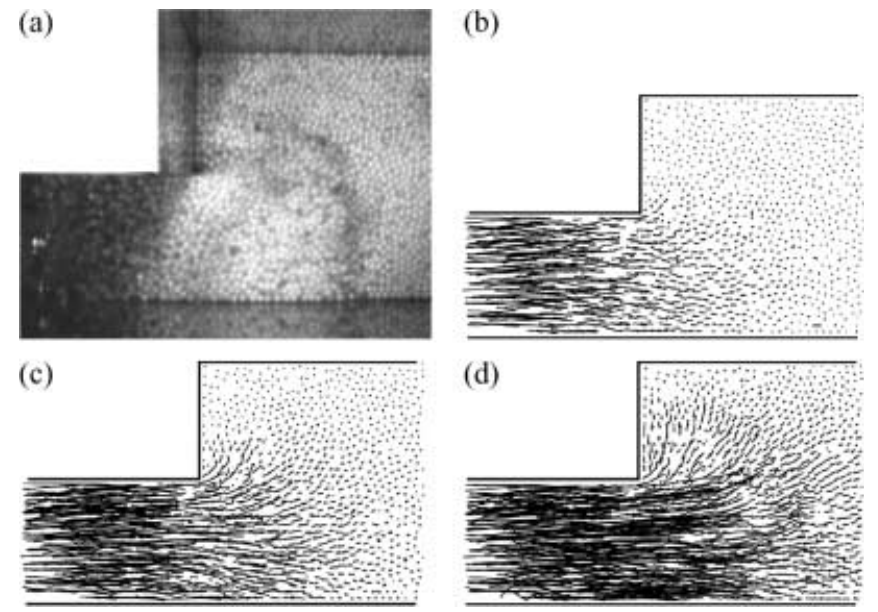

Fig. 17a-d. Top-view analysis for the dam-break experiments: a original image frame at time $t=0.35$; b granular trajectories tracked until $t=0.19 \mathrm{~s} ; \mathrm{c} t=0.27 \mathrm{~s} ; \mathbf{d} t=0.35 \mathrm{~s}$. Dimensions are given by the flume width, equal to $10 \mathrm{~cm}$ before the enlargement, and $20 \mathrm{~cm}$ beyond applications. Specifically, the Voronoï methods were used to derive granular velocities, concentrations, temperatures, and trajectories. They were able to capture rapidly sheared, densely packed dispersions of fluctuating grains in a fully automated way. This makes it possible to obtain long time trajectories and statistically significant measurement samples from experiments, which could otherwise only be obtained from computational simulations. While the methods are presently limited to rather idealized situations with well-sorted artificial grains, work is presently in progress to extend them to more complex granular materials. To obtain three-dimensional measurements, stereoscopic extensions are also contemplated.

\section{Appendix 1: Particle-positioning algorithm}

For the application of Voronoï imaging techniques to dense granular flows, it is desirable to obtain particle positions (and above all, displacements) accurate to a small fraction of the grain diameter. Starting with images for which a particle diameter typically spans between 5 and 20 pixels, the following algorithm has been found to yield good results, producing an estimated root-mean-square error of the order of 0.25 pixel.

The images considered feature white, nearly spherical particles, which contrast well against the darker surrounding fluid. With reference to Fig. 18, the first step of the positioning algorithm is to convolute the images with a Mexican hat filter. Let $g(x, y)$ denote an image in the form of a grey-level map, and $\boldsymbol{G}=g_{i, j}$ the corresponding greylevel matrix of size $n_{x} \times n_{y}$. A smoothing operator is first applied in the form of a binomial filter of order $m=2 r$ (Jähne 1995):

$\boldsymbol{G}^{\prime}=\left[\boldsymbol{B}_{y}^{(m)}\left(\boldsymbol{B}_{x}^{(m)} \boldsymbol{G}\right)^{\mathrm{T}}\right]^{\mathrm{T}}$

where $\boldsymbol{B}_{x}^{(m)}\left(\right.$ resp. $\left.\boldsymbol{B}_{y}^{(m)}\right)$ is a band diagonal matrix of size $n_{x} \times n_{x}\left(\right.$ resp. $\left.n_{y} \times n_{y}\right)$ and bandwidth $m+1=2 r+1$, having non-zero terms

$b_{i, i+k}=\frac{1}{2^{m}} \frac{m !}{(r-k) !(r+k) !}(k=-r, \ldots, r)$

The size $m$ of the binomial filter is chosen as the even integer closest to $1 / 3 d^{2}$, where $d$ is the pixel diameter of the particles. The following Laplace operator (Jähne 1995) is then applied:

$$
\boldsymbol{G}^{\prime \prime}=\boldsymbol{G}^{\prime}-\left[\boldsymbol{B}_{y}^{(2)}\left(\boldsymbol{B}_{x}^{(2)} \mathbf{G}^{\prime}\right)^{\mathrm{T}}\right]^{\mathrm{T}}
$$

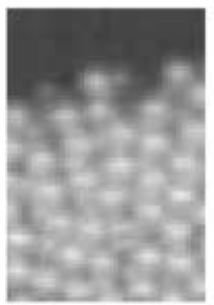

(a)

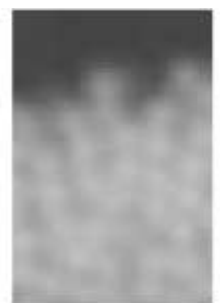

(b)

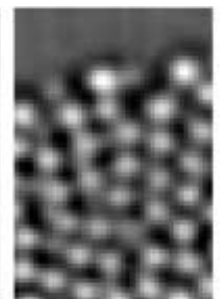

(c)

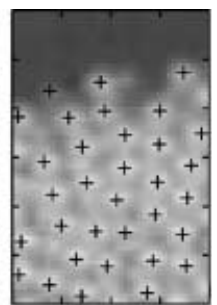

(d)
Fig. 18a-d. Particle-positioning algorithm: a close-up of an original image frame; $\mathbf{b}$ after binomial smoothing operator; $\mathbf{c}$ after Laplace operator; $\mathbf{d}$ obtained particle positions 
where $\boldsymbol{B}_{x}^{(2)}$ and $\boldsymbol{B}_{y}^{(2)}$ are defined as in (22). The specific form of Laplacian (24) is chosen to reduce the anisotropy induced by the orientation of the pixel array (Jähne 1995). The conjunction of the two operators is equivalent to a convolution of the original image with a Mexican hat filter. It has the net effect of highlighting grain centroids, characterized by a radially symmetric pattern of brightness at the centre and darkness one radius away. The discrete positions of particle centroids are then obtained as local maxima $g_{i(k), j(k)}^{\prime \prime}$ of the filtered image $G^{\prime \prime}$. Finally, the positions are interpolated to subpixel accuracy according to

$$
\begin{aligned}
& x_{k}=x_{i(k)}+\frac{\frac{1}{2}\left(g_{i(k)-1, j(k)}^{\prime \prime}-g_{i(k)+1, j(k)}^{\prime \prime}\right)}{g_{i(k)-1, j(k)}^{\prime \prime}-2 g_{i(k), j(k)}^{\prime \prime}+g_{i(k)+1, j(k)}^{\prime \prime}} \Delta x \\
& y_{k}=y_{j(k)}+\frac{\frac{1}{2}\left(g_{i(k), j(k)-1}^{\prime \prime}-g_{i(k), j(k)+1}^{\prime \prime}\right)}{g_{i(k), j(k)-1}^{\prime \prime}-2 g_{i(k), j(k)}^{\prime \prime}+g_{i(k), j(k)+1}^{\prime \prime}} \Delta y
\end{aligned}
$$

obtained by fitting parabolas to the grey levels along the $x$ and $y$-directions.

\section{Appendix 2: Visibility of individual particles in a random dispersion}

In the dilute case, reasoning in terms of Poisson dispersions can be used to derive a relationship between volumetric concentration $\phi$ and the feature-point density $\eta$ observed on two-dimensional images. With reference to Fig. 19, consider a set of spherical particles of diameter $d$, randomly dispersed inside a sheet of thickness $\Delta z$. If the dispersion is dilute, one can consider in first approximation that the particle positions are uncorrelated and governed by a homogeneous Poisson process of intensity $n=\phi /\left(\pi d^{3} / 6\right)$. Assuming that a particle is seen if its centre is not hidden from view by another particle, the probability that a particle is visible depends on its distance $z$ from the side wall and is given by

$$
\begin{gathered}
\operatorname{prob}\left[\text { no particle inside cylinder of volume } \frac{\pi d^{2} z}{2}\right] \\
\quad=\exp \left(-n \frac{\pi d^{2} z}{4}\right)
\end{gathered}
$$
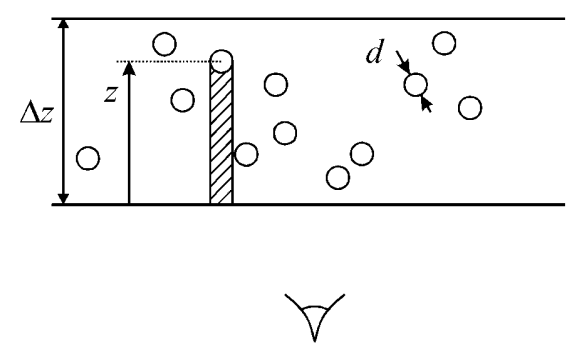

Fig. 19. Relation between the volumetric concentration $\phi$ and the projected two-dimensional feature-point density $\eta$. A particle is visible if no other particle centre is present in the cross-hatched cylinder
Since the distance to the wall $z$ of a particle picked at random is uniformly distributed over interval $[0, \Delta z]$, one obtains by integration the result (12), i.e.,

$$
\eta=\frac{4}{\pi d^{2}}\left[1-\exp \left(-\frac{3}{2} \frac{\phi \Delta z}{d}\right)\right]
$$

where $\eta$ is the expected density of feature-points (i.e., the number of feature-points per unit image surface) observed through the side wall. The reasoning above is rather idealized (illumination is taken for granted and the simplest possible projection onto the image plane is assumed). The important point is that, in any hypothesis, the sensitivity of $\eta$ with $\phi$ rapidly decays with a growth in the ratio $\phi \Delta z / d$. In particular, a direct proportional relation between $\eta$ and $\phi$ is only obtained in the very dilute state, far from the concentrations encountered in the granular flow applications of the present work.

\section{References}

Adrian RJ (1991) Particle-imaging techniques for experimental fluid mechanics. Annu Rev Fluid Mech 23: 261-304

Ahuja N (1982) Dot pattern processing using Voronoï neighborhoods. IEEE Trans Pattern Anal Machine Intell 4: 336-343

Albers G; Guibas LJ; Mitchell JSB; Roos T (1998) Voronoï diagrams of moving points. Int J Comput Geom Appl 8: 365-379

Allen SM; Thomas EL (1999) The structure of materials. Wiley, New York

Armanini A; Fraccarollo L; Guarino L; Martino R; Bin Y (2000) Experimental analysis of the general features of uniform debris flow over a loose bed. Second Int Conf on Debris-Flow Hazard Mitigation, Taipei, August, pp 327-334

Bachalo WD (1994) Experimental methods in multiphase flows. Int J Multiphase Flow 20: 261-295

Batchelor GK (1988) A new theory of the instability of a uniform fluidized bed. J Fluid Mech 193: 75-110

Bennett SJ; Best JL (1995) Particle size and velocity discrimination in a sediment-laden turbulent flow using phase Doppler anemometry. J Fluid Eng 117: 505-511

Bernardeau F; van de Weygaert R (1996) A new method for accurate estimation of velocity field statistics. Mon Not Roy Astr Soc 279: 693-711

Braun J; Sambridge M (1995) A numerical method for solving differential equations on highly irregular evolving grids. Nature 376: 655-660

Brookner E (1998) Tracking and Kalman filtering made easy. Wiley, New York

Campbell CS (1990) Rapid granular flows. Annu Rev Fluid Mech 22: $57-92$

Capart H (2000) Dam-break induced geomorphic flows and the transition from solid- to fluid-like behaviour across evolving interfaces. PhD thesis, UCL, Belgium

Capart H; Liu HH; Van Crombrugghe X; Young DL (1997) Digital imaging characterization of the kinematics of water-sediment interaction. Water Air Soil Pollut 99: 173-177

Capart H; Young DL (1998) Formation of a jump by the dambreak wave over a granular bed. J Fluid Mech 372: 165-187

Capart H; Fraccarollo L; Guarino L; Armanini A; Zech Y (2000) Granular temperature behaviour of loose-bed debris flows. Second Int Conf on Debris-Flow Hazard Mitigation, Taipei, August, pp 361-368

Capart H; Young DL; Zech Y (2001) Dam-break induced debris flow. In: McCaffrey B, Kneller B, Peakall J (eds) Particulate gravity currents, Spec. Publ. Int. Assoc. Sediment. vol. 31. Blackwell Science, Oxford 
Chaouki J; Larachi F; Dudukovic MP (1997) Noninvasive tomographic and velocimetric monitoring of multiphase flows. Ind Eng Chem Res 36: 4476-4503

Costa JE; Schuster RL (1988) The formation and failure of natural dams. Bull Geol Soc Am 100: 1054-1068

Cui MM; Adrian RJ (1997) Refractive index matching and marking methods for highly concentrated solid-liquid flows. Exp Fluids 22: 261-264

Dave RN; Rosato A; Fischer IS (1999) Nonintrusive particle tracking system for particulate flows and vibrated granular beds. Particulate Sci Tech 17: 125-139

Drake TG (1991) Granular flow: Physical experiments and their implications for microstructural theories. J Fluid Mech 225: 121-152

Duda RO; Hart PE (1973) Pattern classification and scene analysis. Wiley-Interscience

Elliott KE; Ahmadi G; Kvasnak W (1998) Couette flows of a granular monolayer - an experimental study. J Non-Newtonian Fluid Mech 74: 89-111

Espanol P (1998) Fluid particle model. Phys Rev E 57: 2930-2948

Fortune S (1987) A sweepline algorithm for Voronoi diagrams. Algorithmica 2: 153-174

Frenkel D; Smit B (1996) Understanding molecular simulation: From algorithms to applications. Academic Press, San Diego

Fujita I; Muste M; Kruger A (1998) Large-scale particle image velocimetry for flow analysis in hydraulic engineering applications. J Hydr Res 36: 397-414

Goodrich MT; Mitchell JSB; Orletsky MW (1999) Approximate geometric pattern matching under rigid motions. IEEE Trans Pattern Anal Machine Intell 21: 371-379

Guarino L (1998) Moto uniforme a superficie libra di misture granulari solido-liquido ad alta concentrazione. BEng thesis, Università degli studi di Trento, Italy

Guler M; Edil TB; Bosscher PJ (1999) Measurement of particle movement in granular soils using image analysis. J Comp Civ Eng 13: 116-122

Haynes ID; Turner JT (1992) Sixth Int Symp on the Application of Laser Techniques to Fluid Mechanics, pp 3.3.1-3.3.6

Hsiau SS; Jang HW (1998) Measurements of velocity fluctuations of granular materials in a shear cell. Exp Therm Fluid Sci 17: 202-209

Huang HT; Fiedler HE; Wang JJ (1993) Limitation and improvement of PIV. Part I: Limitation of conventional techniques due to deformation of particle image patterns. Exp Fluids 15: 168-174

Jähne B (1995) Digital image processing. Springer, Berlin Heidelberg New York

Jain R; Kasturi R; Schunck BG (1995) Machine vision. McGrawHill, New York

Jenkins JT; Hanes DM (1998) Collisional sheet-flow of sediment driven by a turbulent fluid. J Fluid Mech 370: 29-52

Kanda T; Miyamoto H; Iwami S (1999) Measurement of suspended solid concentration by using image processing technique. XXVII IAHR Biennal Congr, Graz, August, p 253

Kalthoff W; Schwarzer S; Herrmann H (1997) An algorithm for the simulation of particulate suspensions with inertia effects. Phys Rev E 56: 2234-2242

Kenkel NC; Hoskins JA; Hoskins WD (1989) Edge effects in the use of area polygons to study competition. Ecology 70: 272-274

Lee HY; Hsu IS (1994) Investigation of saltating particle motions. J Hydr Engrg 120: 831-845

Liu KF; Lee FC; Tsai HP (1997) The flow field and impact force on a debris dam. First Int Conf on Debris-flow Hazard Mitigation, San Francisco, August, pp 737-743

Lloyd PM; Ball DJ; Stansby PK (1995) Unsteady surface-velocity field measurement using particle tracking velocimetry. J Hydr Res 33: 519-533
Louge MY; Jenkins JT (1997) Microgravity segregation in binary mixtures of inelastic spheres driven by velocity fluctuation gradients. In: Chang CS, Misra A, Liang RY, Babic M (eds) Mechanics of deformation and flow of particulate materials. ASCE, Reston, USA, pp 370-379

Malik NA; Dracos T; Papantoniou D (1993) Particle tracking velocimetry in three-dimensional flows. Part II: Particle tracking. Exp Fluids 15: 279-294

Marcelpoil R; Usson Y (1992) Methods for the study of cellular sociology: Voronoï diagrams and parametrization of the spatial relationship. J Theor Biol 154: 359-369

Nakagawa M; Altobelli SA; Caprihan A; Fukushima E; Jeong EK (1993) Noninvasive measurements of granular flows by magnetic-resonance-imaging. Exp Fluids 16: 54-60

Natarajan VVR; Hunt ML; Taylor ED (1995) Local measurements of velocity fluctuations and diffusion coefficients for a granular material flow. J Fluid Mech 304: 1-25

Okabe A; Boots B; Sugihara K (1992) Spatial tesselations: Concepts and applications of Voronoï diagrams. Wiley, New York

Preparata FP; Shamos MI (1985) Computational geometry: An introduction. Springer, Berlin Heidelberg New York

Richardson JF; Zaki W (1954) Sedimentation and fluidization: Part I. Trans Instn Chem Engrs 32: 35-53

Ruan XD; Song XQ; Fujio Y (1999) Research on the algorithm of binary image cross-correlation for unsteady flow field measurement. Acta Mechanica Sinica 15: 32-38

Savage SB; McKeown S (1983) Shear stresses developed during rapid shear of concentrated suspensions of large spherical particles between concentric cylinders. J Fluid Mech 127: 453-472

Senechal M (1995) Quasicrystals and geometry. Cambridge Univ Press, Cambridge

Sethi IK; Jain R (1987) Finding trajectories of feature points in a monocular image sequence. IEEE Trans Pattern Anal Machine Intell 9: 56-73

Sibson R (1981) A brief description of natural neighbour interpolation. In: Barnett $C$ (ed) Interpreting multivariate data, Wiley, New York, pp 21-36

Song XQ; Yamamoto F; Iguchi M; Murai Y (1996) A new crosscorrelation algorithm for PIV based on Delaunay tesselation. J Visual Soc Japan 16: 19-22

Song XQ; Yamamoto F; Iguchi M; Murai Y (1999) A new tracking algorithm of PIV and removal of spurious vectors using Delaunay tesselation. Exp Fluids 26: 371-380

Takahashi T (1991) Debris flow. IAHR Monograph. Balkema, Rotterdam

Uddin MS; Inaba H; Itakura Y; Kasahara M (1998) Estimation of the surface velocity of debris flow with computer-based spatial filtering. Appl Opt 37: 6234-6239

Ushijima S; Tanaka N (1996) Three-dimensional particle tracking velocimetry with laser-light sheet scannings. J Fluid Eng 118: 352-357

Veber P; Dahl J; Hermansson R (1997) Study of the phenomena affecting the accuracy of a video-based particle tracking velocimetry technique. Exp Fluids 22: 482-488

Wallet F; Dussert C (1998) Comparison of spatial point pattern and process characterization methods. Europhys Lett 42: 493498

Wildman RD; Huntley JM; Hansen JP (1999) Self-diffusion of grains in a two-dimensional vibrofluidized bed. Phys Rev E 60: 7066-7075

Willert CE; Gharib M (1991) Digital particle image velocimetry. Exp Fluids 10: 181-193

Zenit R; Hunt ML; Brennen CE (1997) Collisional particle pressure measurements in solid-liquid flows. J Fluid Mech 353: 261-283 